\title{
ON IIEB AND THIRRING TYPE DISCRETE INEQUALITIES
}

\author{
B.G. PACHPATTE
}

\begin{abstract}
Discrete inequalities of the Lieb and Thirring type involving functions of several independent variables and their forward differences are established. The proofs given here are elementary and the results established provide new estimates on these types of inequalities.
\end{abstract}

\section{Introduction}

In [4] Lieb and Thirring have given the following interesting inequality.

Let $u_{r}(r=1, \cdots, M)$ be a finite family of functions in $H^{1}\left(R^{n}\right)$ which are orthonormal in $L^{2}\left(R^{n}\right)$ and let $p$ be a constant satisfying $\max \left(1, \frac{n}{2}\right)<p \leq 1+\left(\frac{n}{2}\right)$. Then

$$
\left[\int_{R^{n}}\left\{\sum_{r=1}^{M} u_{r}(x)^{2}\right\}^{\frac{p}{p-1}} d x\right]^{\frac{2(p-1)}{n}} \leq k_{0} \sum_{r=1}^{M} \int_{R^{n}} \sum_{i=1}^{n}\left\{\frac{\partial}{\partial x_{i}} u_{r}(x)\right\}^{2} d x
$$

where $k_{0}=k_{0}(n, p)$ is the constant independent of $M$ and $u_{r}$.

The inequality (1) is an extension of the well known Sobolev-Gagliardo-Nirenberg inequality (see, $[2,5,8]$ ). A number of interesting generalizations of the inequality (1) which have applications in the study of the dimension of attractors associated with dissipative parabolic equations are recently given by Ghidaglia, Marion and Temam in [3]. Aside from the applications, inequalities like (1) are of interest in their own right and we believe that the discrete inequalities of the type (1) will be a new addition to the literature on such inequalities. The main purpose of the present paper is to establish some new discrete inequalities of the type (1) involving functions of several independent variables and their forward differences. The method used in the proofs is elementary and our results provide new estimates on these types of discrete inequalities.

\section{Statement of results}

In what follows, we let $R$ be the set of real numbers and $N=\{1,2, \cdots\}$. For $\mathscr{x}=\left(x_{1}, \ldots, x_{n}\right) \in N^{n}$ and $z(x): N^{n} \rightarrow R$, we define the forward difference operators as 
follows:

$$
\begin{gathered}
\triangle_{1} z\left(x_{1}, \ldots, x_{n}\right)=z\left(x_{1}+1, x_{2}, \ldots, x_{n}\right)-z\left(x_{1}, \ldots, x_{n}\right), \\
\vdots \\
\triangle_{n} z\left(x_{1}, \ldots, x_{n}\right)=z\left(x_{1}, \ldots, x_{n-1}, x_{n}+1\right)-z\left(x_{1}, \ldots, x_{n}\right) .
\end{gathered}
$$

The notation $\triangle_{i} z\left(x_{1}, \ldots, y_{i}, \ldots, x_{n}\right)$ for $i=1, \cdots, n$ we mean for $i=1$ it is $\triangle_{1} z\left(y_{1}, x_{2}\right.$, $\left.\ldots, x_{n}\right)$ and so on and for $i=n$ it is $\triangle_{n} z\left(x_{1}, \ldots, x_{n-1}, y_{n}\right)$. Let $B$ be a bounded domain in $N^{n}$ with $n \geq 1$ defined by $B=\{x: \underline{1} \leq x \leq a+\underline{1}\}$ where $\underline{1}=(1, \cdots, 1) \in N^{n}, x=$ $\left(x_{1}, \ldots, x_{n}\right) \in N^{n}, a=\left(a_{1}, \ldots, a_{n}\right) \in N^{n}$. We define $\alpha=\max \left\{a_{1}, \ldots, a_{n}\right\}$. We denote by $F(B)$ the class of functions $z(x): B \rightarrow R$ for which $\triangle_{i} z\left(x_{1}, \ldots, y_{i}, \ldots, x_{n}\right)$ exist and such that

$$
\begin{aligned}
z\left(1, x_{2}, \ldots, x_{n}\right) & =z\left(x_{1}, 1, x_{3}, \ldots, x_{n}\right)=\cdots=z\left(x_{1}, \ldots, x_{n-1}, 1\right)=0 \\
z\left(a_{1}+1, x_{2}, \ldots, x_{n}\right) & =z\left(x_{1}, a_{2}+1, x_{3}, \ldots, x_{n}\right)=\cdots=z\left(x_{1}, \ldots, x_{n-1}, a_{n}+1\right)=0
\end{aligned}
$$

For $z(x): B \rightarrow R$ we use the following notation

$$
\sum_{B} z(y)=\sum_{y_{1}=1}^{a_{1}} \cdots \sum_{y_{n}=1}^{a_{n}} z\left(y_{1}, \ldots, y_{n}\right)
$$

and also use the customary convention

$$
\sum_{y_{1}=a_{1}}^{a_{1}-1} z\left(y_{1}, x_{2}, \ldots, x_{n}\right)=0, \ldots, \sum_{y_{n}=a_{n}}^{a_{n}-1} z\left(x_{1}, \ldots, x_{n-1}, y_{n}\right)=0 .
$$

Our main result is established in the following theorem.

Theorem 1. Let $u_{r} \in F(B)$ for $r=1, \cdots, M$ and let $m \geq 1$ and $p \geq 2$ be real constants. Then

$$
\left[\sum_{B}\left\{\sum_{r=1}^{M}\left|u_{r}(y)\right|^{2}\right\}^{\frac{p}{p-1}}\right]^{\frac{2 m(p-1)}{p}} \leq k_{1} \sum_{r=1}^{M} \sum_{B} \sum_{i=1}^{n}\left|\triangle_{i} u_{r}(y)\right|^{4 m},
$$

where

$$
k_{1}=\frac{1}{n}\left(\frac{1}{4}\right)^{2 m} M^{2 m-1} \alpha^{\frac{p(4 m+2 n m-n)-2 n m}{p}}
$$

Remark 1 . If we take $m=1$ in (2), then we get the inequality analogous to the discrete version of the inequality (1). On taking $u_{r}(x)=u(x)$ for $r=1, \cdots, M$ and $m=1, p=2$ in (2) we get the following inequality

$$
\sum_{B}|u(x)|^{4} \leq \frac{1}{n}\left(\frac{\alpha}{2}\right)^{4} \sum_{B} \sum_{i=1}^{n}\left|\triangle_{i} u(y)\right|^{4}
$$


For different versions of inequalities of the type (2)-(3), see [6,7].

A slight variant of the inequality (2) motivated by the Dubinskii's inequalities [1] is embodied in the following theorem.

Theorem 2. Let $u_{r}, m, p$ be as defined in Theorem 1. Then

$$
\begin{aligned}
& {\left[\sum_{B}\left\{\sum_{r=1}^{M}\left|u_{r}(y)\right|^{2}\right\}^{\frac{p}{p-1}}\right]^{\frac{2 m(p-1)}{p}} } \\
\leq & k_{2} \sum_{r=1}^{M} \sum_{B} \sum_{i=1}^{n}\left|\Delta_{i} u_{r}(y)\right|^{4 m}+k_{3} \sum_{r=1}^{M} \sum_{B} \sum_{i=1}^{n}\left|u_{r}(y)\right|^{2 m}\left|\Delta_{i} u_{r}(y)\right|^{2 m},
\end{aligned}
$$

where

$$
k_{2}=\frac{1}{2 n} M^{2 m-1} \alpha^{\frac{p(2 m+2 n m-n)-2 n m}{p}}, \quad k_{3}=2^{2 m} k_{2}
$$

Remark 2. In the special case when $m=1, p=2$ and $u_{r}(x)=u(x)$ for $r=$ $1, \cdots, M$, inequality (4) reduces to

$$
\sum_{B}|u(y)|^{4} \leq \frac{\alpha^{2}}{2 n} \sum_{B} \sum_{i=1}^{n}\left|\triangle_{i} u(y)\right|^{4}+\frac{2 \alpha^{2}}{n} \sum_{B} \sum_{i=1}^{n}|u(y)|^{2}\left|\triangle_{i} u(y)\right|^{2} .
$$

It is interesting to note that the inequality (5) is analogous to the discrete analogue of the Sobolev's inequality [2]. However the bound obtained in (5) is not exactly the discrete analogue of the bound involved on the right side of the Sobolev's inequality (see, [5,p.1]).

\section{Proofs of Theorems 1 and 2}

Since $u_{r} \in F(B)$, we have the following identities:

$$
\begin{aligned}
& n u_{r}(x)=\sum_{i=1}^{n}\left\{\sum_{y_{i}=1}^{x_{i}-1} \triangle_{i} u_{r}\left(x_{1}, \ldots, y_{i}, \ldots, x_{n}\right)\right\} \\
& n u_{r}(x)=-\sum_{i=1}^{n}\left\{\sum_{y_{i}=x_{i}}^{a_{i}} \triangle_{i} u_{r}\left(x_{1}, \ldots, y_{i}, \ldots, x_{n}\right)\right\}
\end{aligned}
$$

for $r=1, \cdots, M$. From (6) and (7) we obtain

$$
\left|u_{r}(x)\right| \leq \frac{1}{2 n} \sum_{i=1}^{n}\left\{\sum_{y_{i}=1}^{a_{i}}\left|\triangle_{i} u_{r}\left(x_{1}, \ldots, y_{i}, \ldots, x_{n}\right)\right|\right\} .
$$

From (8) and on using the elementary inequality (see, [5, p.5])

$$
\left\{\sum_{i=1}^{n} c_{i}\right\}^{k} \leq n^{k-1} \sum_{i=1}^{n} c_{i}^{k}
$$


where $c_{1}, \ldots, c_{n} \geq 0$ reals $k \geq 1$, Schwarz inequality and the definition of $\alpha$, we obtain

$$
\begin{aligned}
\left|u_{r}(x)\right|^{2} & \leq\left(\frac{1}{2 n}\right)^{2}\left[\sum_{i=1}^{n}\left\{\sum_{y_{i}=1}^{a_{i}}\left|\triangle_{i} u_{r}\left(x_{1}, \ldots, y_{i}, \ldots, x_{n}\right)\right|\right\}\right]^{2} \\
& \leq\left(\frac{1}{2 n}\right)^{2} n \sum_{i=1}^{n}\left\{\sum_{y_{i}=1}^{a_{i}}\left|\triangle_{i} u_{r}\left(x_{1}, \ldots, y_{i}, \ldots, x_{n}\right)\right|\right\}^{2} \\
& \leq\left(\frac{\alpha}{4 n}\right) \sum_{i=1}^{n}\left\{\sum_{y_{i}=1}^{a_{i}}\left|\triangle_{i} u_{r}\left(x_{1}, \ldots, y_{i}, \ldots, x_{n}\right)\right|^{2}\right\} .
\end{aligned}
$$
From (10) and on using inequality (9) repeatedly, Hölder's inequality with indices $p, \frac{p}{p-1}$
and the definition of $\alpha$, we obtain

$$
\begin{aligned}
\left\{\sum_{r=1}^{M}\left|u_{r}(x)\right|^{2}\right\}^{\frac{p}{p-1} \leq} & \left(\frac{\alpha}{4 n}\right)^{\frac{p}{p-1}}(m n) \frac{p}{p-1}-1 \\
& \cdot \sum_{r=1}^{M}\left\{\sum_{i=1}^{n}\left\{\sum_{y_{i}=1}^{a_{i}}\left|\triangle_{i} u_{r}\left(x_{1}, \ldots, y_{i}, \ldots, x_{n}\right)\right|^{2}\right\} \frac{p}{p-1}\right\} \\
\leq & \left(\frac{\alpha}{4 n}\right)^{\frac{p}{p-1}}(M n) \frac{1}{p-1} \frac{1}{p-1} \\
& \cdot \sum_{r=1}^{M}\left\{\sum_{i=1}^{n}\left\{\sum_{y_{i}=1}^{a_{i}}\left|\triangle_{i} u_{r}\left(x_{1}, \ldots, y_{i}, \ldots, x_{n}\right)\right| \frac{2 p}{p-1}\right\}\right\} .
\end{aligned}
$$

Setting $x_{i}=y_{i}(i=1, \cdots, n)$ in (11) and taking the sum over both sides of (11) with respect to $y_{1}, \ldots, y_{n}$ on $B$ and using the definition of $\alpha$, we have

$$
\begin{aligned}
\sum_{B}\left\{\sum_{r=1}^{M}\left|u_{r}(y)\right|^{2}\right\}^{\frac{p}{p-1}} \leq & \left(\frac{\alpha}{4 n}\right)^{\frac{p}{p-1}}(\operatorname{Mn\alpha })^{\frac{1}{p-1}} \alpha \\
& \cdot \sum_{r=1}^{M}\left\{\sum_{i=1}^{n}\left\{\sum_{B}\left|\Delta_{i} u_{r}(y)\right|^{\frac{2 p}{p-1}}\right\}\right\} .
\end{aligned}
$$

From (12) and on using the inequality (9) repeatedly, Hölder's inequality with indices $\frac{2 m(p-1)}{p}, \frac{2 m(p-1)}{2 m(p-1)-p}$ and the definition of $\alpha$, we obtain

$$
\left[\sum_{B}\left\{\sum_{r=1}^{M}\left|u_{r}(y)\right|^{2}\right\}^{\frac{p}{p-1}}\right]^{\frac{2 m(p-1)}{p}}
$$




$$
\begin{aligned}
\leq & \left\{\left(\frac{\alpha}{4 n}\right)^{\frac{p}{p-1}}(M n \alpha) \frac{1}{p-1} \alpha\right\} \frac{2 m(p-1)}{p}(M n) \frac{2 m(p-1)}{p}-1 \\
& \times \sum_{r=1}^{M}\left\{\sum_{i=1}^{n}\left\{\sum_{B} \mid \Delta_{i} u_{r}(y) \frac{2 p}{p-1}\right\} \frac{2 m(p-1)}{p}\right\} \\
\leq & \left\{\left(\frac{\alpha}{4 n}\right) \frac{p}{p-1}(M n \alpha) \frac{1}{p-1} \alpha\right\} \frac{2 m(p-1)}{p}(M n) \frac{2 m(p-1)}{p}-1 \\
& \times\left(\alpha^{n}\right) \frac{2 m(p-1)-p}{p} \sum_{r=1}^{M}\left\{\sum_{i=1}^{n}\left\{\sum_{B}\left|\triangle_{i} u_{r}(y)\right|^{4 m}\right\}\right\} \\
= & K_{1} \sum_{r=1}^{M} \sum_{B} \sum_{i=1}^{n}\left|\triangle_{i} u_{r}(y)\right|^{4 m} .
\end{aligned}
$$

This completes the proof of Theorem 1 .

From the assumptions on the functions $u_{r}(x)$ in Theorem 2, we have the following identities:

$$
\begin{aligned}
& n u_{r}^{2}(x)=\sum_{i=1}^{n}\left\{\sum_{y_{i}=1}^{x_{i}-1} \triangle_{i} u_{r}^{2}\left(x_{1}, \ldots, y_{i}, \ldots, x_{n}\right)\right\} \\
& n u_{r}^{2}(x)=-\sum_{i=1}^{n}\left\{\sum_{y_{i}=x_{i}}^{a_{i}} \triangle_{i} u_{r}^{2}\left(x_{1}, \ldots, y_{i}, \ldots, x_{n}\right)\right\}
\end{aligned}
$$

for $r=1, \cdots, M$. From (14) and (15) we observe that

$$
\begin{aligned}
\left|u_{r}(x)\right|^{2} \leq & \frac{1}{2 n} \sum_{i=1}^{n}\left\{\sum_{y_{i}=1}^{a_{i}}\left|\triangle_{i} u_{r}^{2}\left(x_{1}, \ldots, y_{i}, \ldots, x_{n}\right)\right|\right\} \\
= & \frac{1}{2 n} \sum_{i=1}^{n}\left\{\sum_{y_{i}=1}^{a_{i}}\left|u_{r}^{2}\left(x_{1}, \ldots, y_{i}+1, \ldots, x_{n}\right)-u_{r}^{2}\left(x_{1}, \ldots, y_{i}, \ldots, x_{n}\right)\right|\right\} \\
= & \frac{1}{2 n} \sum_{i=1}^{n}\left\{\sum_{y_{i}=1}^{a_{i}} \mid \triangle_{i} u_{r}\left(x_{1}, \ldots, y_{i}, \ldots, x_{n}\right)\right. \\
& \left.\cdot\left\{u_{r}\left(x_{1}, \ldots, y_{i}+1, \ldots, x_{n}\right)+u_{r}\left(x_{1}, \ldots, y_{i}, \ldots, x_{n}\right)\right\} \mid\right\} \\
= & \frac{1}{2 n} \sum_{i=1}^{n}\left\{\sum_{y_{i}=1}^{a_{i}} \mid \triangle_{i} u_{r}\left(x_{1}, \ldots, y_{i}, \ldots, x_{n}\right)\right. \\
& \left.\cdot\left\{\triangle_{i} u_{r}\left(x_{1}, \ldots, y_{i}, \ldots, x_{n}\right)+2 u_{r}\left(x_{1}, \ldots, y_{i}, \ldots, x_{n}\right)\right\} \mid\right\} \\
= & \frac{1}{2 n} \sum_{i=1}^{n}\left\{\sum_{y_{i}=1}^{a_{i}} \mid\left\{\triangle_{i} u_{r}\left(x_{1}, \ldots, y_{i}, \ldots, x_{n}\right)\right\}^{2}\right. \\
& \left.+2 u_{r}\left(x_{1}, \ldots, y_{i}, \ldots, x_{n}\right) \triangle_{i} u_{r}\left(x_{1}, \ldots, y_{i}, \ldots, x_{n}\right) \mid\right\} .
\end{aligned}
$$


From (16) and on using inequality (9) repeatedly, Hölder's inequality with indices $p, \frac{p}{p-1}$ and the definition of $\alpha$, we obtain

$$
\begin{aligned}
& \left\{\sum_{r=1}^{M}\left|u_{r}(x)\right|^{2}\right\}^{\frac{p}{p-1}} \\
\leq & \left(\frac{1}{2 n}\right)^{\frac{p}{p-1}}(M n) \frac{p}{p-1}-1 \sum_{r=1}^{M}\left\{\sum _ { i = 1 } ^ { n } \left\{\sum_{y_{i}=1}^{a_{i}} \mid\left\{\triangle_{i} u_{r}\left(x_{1}, \ldots, y_{i}, \ldots, x_{n}\right)\right\}^{2}\right.\right. \\
& \left.\left.+2 u_{r}\left(x_{1}, \ldots, y_{i}, \ldots, x_{n}\right) \triangle_{i} u_{r}\left(x_{1}, \ldots, y_{i}, \ldots, x_{n}\right) \mid\right\} \frac{p}{p-1}\right\} \\
\leq & \left(\frac{1}{2 n}\right) \frac{p}{p-1}(M n) \frac{p}{p-1}-1 \frac{1}{p-1} \\
& \sum_{r=1}^{M}\left\{\sum _ { i = 1 } ^ { n } \left\{\sum_{y_{i}=1}^{a_{i}} \mid\left\{\triangle_{i} u_{r}\left(x_{1}, \ldots, y_{i}, \ldots, x_{n}\right)\right\}^{2}\right.\right. \\
& \left.\left.+2 u_{r}\left(x_{1}, \ldots, y_{i}, \ldots, x_{n}\right) \triangle_{i} u_{r}\left(x_{1}, \ldots, y_{i}, \ldots, x_{n}\right) \frac{p}{\left.\right|^{p-1}}\right\}\right\} .
\end{aligned}
$$

Setting $x_{i}=y_{i}(i=1, \cdots, n)$ in (17) and taking the sum over both sides of (17) with respect to $y_{1}, \ldots, y_{n}$ on $B$ and using the definition of $\alpha$, we have

$$
\begin{aligned}
& \sum_{B}\left\{\sum_{r=1}^{M}\left|u_{r}(y)\right|^{2}\right\}^{\frac{p}{p-1}} \\
\leq & \left(\frac{1}{2 n}\right)^{\frac{p}{p-1}}(M n \alpha)^{\frac{1}{p-1}} \alpha \sum_{r=1}^{M}\left\{\sum_{i=1}^{n}\left\{\sum_{B}\left|\left\{\triangle_{i} u_{r}(y)\right\}^{2}+2 u_{r}(y) \triangle_{i} u_{r}(y)\right| \frac{p}{p-1}\right\}\right\} .
\end{aligned}
$$

From (18) and on using the inequality (9) repeatedly, Hölder's inequality with indices $\frac{2 m(p-1)}{p}, \frac{2 m(p-1)}{2 m(p-1)-p}$ and the definition of $\alpha$, we have

$$
\begin{aligned}
& {\left[\sum_{B}\left\{\sum_{r=1}^{M}\left|u_{r}(y)\right|^{2}\right\}^{\frac{p}{p-1}}\right]^{\frac{2 m(p-1)}{p}} } \\
\leq & \left\{\left(\frac{1}{2 n}\right) \frac{p}{p-1}(M n \alpha) \frac{1}{p-1} \alpha\right\} \frac{2 m(p-1)}{p}(M n) \frac{2 m(p-1)}{p}-1 \\
& \sum_{r=1}^{M}\left\{\sum_{i=1}^{n}\left\{\sum_{B} \mid\left\{\triangle_{i} u_{r}(y)\right\}^{2}+2 u_{r}(y) \triangle_{i} u_{r}(y) \frac{p}{p-1}\right\}^{\frac{2 m(p-1)}{p}}\right\}
\end{aligned}
$$




$$
\begin{aligned}
\leq & \left\{\left(\frac{1}{2 n}\right)^{\frac{p}{p-1}}(M n \alpha) \frac{1}{p-1} \alpha\right\}^{\frac{2 m(p-1)}{p}}(M n) \frac{2 m(p-1)}{p}-1 \\
& \cdot\left(\alpha^{n}\right) \frac{2 m(p-1)-p}{p} \sum_{r=1}^{M}\left\{\sum_{i=1}^{n}\left\{\sum_{B}\left|\left\{\triangle_{i} u_{r}(y)\right\}^{2}+2 u_{r}(y) \triangle_{i} u_{r}(y)\right|^{2 m}\right\}\right\} \\
\leq & \left(\frac{1}{2 n}\right)^{2 m}(M n \alpha) \frac{2 m}{p} \frac{2 m(p-1)}{p}(M n) \frac{2 m(p-1)-p}{p} \\
& \cdot\left(\alpha^{n}\right) \frac{2 m(p-1)-p}{p} \sum_{r=1}^{M}\left\{\sum _ { i = 1 } ^ { n } \left\{\sum _ { B } 2 ^ { 2 m - 1 } \left[\left|\triangle_{i} u_{r}(y)\right|^{4 m}\right.\right.\right. \\
& \left.\left.\left.+2^{2 m}\left|u_{r}(y)\right|^{2 m}\left|\triangle_{i} u_{r}(y)\right|^{2 m}\right]\right\}\right\} \\
= & K_{2} \sum_{r=1}^{M} \sum_{B} \sum_{i=1}^{n}\left|\triangle_{i} u_{r}(y)\right|^{4 m}+k_{3} \sum_{r=1}^{M} \sum_{B} \sum_{i=1}^{n}\left|u_{r}(y)\right|^{2 m}\left|\triangle_{i} u_{r}(y)\right|^{2 m} .
\end{aligned}
$$

This is the required inequality in (4) and the proof of Theorem 2 is complete.

Remark 3. We note that the hypotheses used on finite family of functions $u_{r}$ ( $r=$ $1, \cdots, M)$ and the constant $p$ in our Theorems 1 and 2 are different from those given by the authors in $[3,4]$. We also note that the constants $k_{1}, k_{2}, k_{3}$ involved in (2) and (4) depends on $n, M, p$ and the size of the domain of definitions of the finite family of functions $u_{r}$, while the constant involved in (1) depends on $n, p$ and independent of $M$ and $u_{r}$.

\section{References}

[1] J.A. Dubinskii, "Some integral inequalities and the solvability of degenerate quasilinear elliptic systems of differential equations," Math. Sb. 64 (1964), 458-480.

[2] A. Friedman, "Partial Differential Equations", New York:Holt, Reinhart and Winston, 1969.

[3] J.M. Ghidaglia, M. Marion and R.Temam, "Generalization of the Sobolev-Lieb-Thirring inequalities and applications to the dimension of attractors," Differential and Integral Equations 1 (1988), 1-21.

[4] E. Lieb and W.Thirring, Inequalities for the moments of the eigenvalues of the Schrödinger Hamiltonian and their relation to Sobolev inequalities, in Studies in Mathematical Physics, E. Lieb, B. Simon and A.Wightman Eds.Princeton Univ.Press, Princeton, 1976.

[5] B.G. Pachpatte, "On Sobolev type inequalities," Proc.Royal Soc.Edinburgh 103A (1986), 1-14.

[6] B.G. Pachpatte, On discrete inequalities of the Poincaré type, Periodica Math. Hungarica 19 (1988), 227-233.

[7] B.G. Pachpatte, "On some new multidimensional discrete inequalities," Tamkang Jour.Math. 17 (1986), 21-29.

[8] P.M. Prenter, "Splines and Variational Methods", Wiley, New York, 1975.

Department of Mathematics and Statistics, Marathwada University, A urangabad 431004, (Maharashtra), INDIA. 